\title{
Feuille de route pour le dossier électronique du patient au cabinet du médecin de famille
}

\section{Heinz Bhend, Marco Zoller}

Groupe de travail SSMG.Informatics
Correspondance:

Dr Heinz Bhend

Oltnerstrasse 9

CH-4663 Aarburg

heinz.bhend@sgam.ch

\section{Contexte}

Comme on le sait, la Suisse est très en retard dans l'utilisation des dossiers électroniques de patients au cabinet médical. Comme les cabinets ambulatoires ne sont pas raccordés au flux d'information et que cette lacune exerce à son tour une influence sur le rôle qu'ils doivent jouer, cette situation peut devenir problématique.

Les résultats de notre dernière enquête [1] montrent que seuls environ $11 \%$ des médecins établis en cabinet privé ont passé entièrement à la documentation électronique. Mais le constat le plus significatif de cette étude est que deux tiers des médecins interrogés n'envisagent clairement pas un tel transfert dans les trois prochaines années. Ce retard est dû essentiellement à l'absence de standards pour les dossiers électroniques de patients. Cette situation empêche, en effet, l'échange et la migration des données et entrave massivement tout changement de fournisseurs de prestations. En lançant une feuille de route (road map) pour les dossiers électroniques de patients, nous nous engageons dans une voie pragmatique pour animer la discussion et esquisser des solutions réalisables.

Comme de nombreux médecins sont dépassés par la question de savoir quels sont les critères à prendre en compte pour évaluer un logiciel, nous présentons ci-après les exigences minimales posées à un système pour être conforme au cabinet médical. Lorsqu'un produit remplit ces exigences dans le cadre d'un test neutre, il obtient le label du groupe de travail SGAM/SSMG.Informatics. En outre, les médecins praticiens ont à leur disposition des listes de contrôle pour évaluer les produits et des ateliers pour planifier le passage de leur cabinet à une entreprise libérée de la paperasserie.

\section{Label SGAM/SSMG.Informatics pour les dossiers électroniques de patients}

L'objectif du label «SGAM/SSMG.Informatics» vise avant tout l'unification des systèmes sur la base d'une série minimale de données, aux fins de permettre à l'avenir l'échange de données entre différents logiciels.

Nous ne nous occupons sciemment que de la partie relative à la documentation clinique en partant du principe que les données administra-

\section{Exemple}

M. Huber déménage de $\mathrm{A}$ à $\mathrm{B}$. Son médecin de famille à son premier domicile, à savoir le Dr Müller, a géré un dossier électronique de patient à son sujet durant les trois dernières années. Les notes, valeurs de la pression sanguine et données d'analyse de laboratoire sont classées sur ce support électronique d'après la date. Les médicaments prescrits, leur changement, le dosage exact et leur intolérance figurent également dans ce dossier. Le Dr Müller a établi une liste des problèmes selon la classification ICPC-2 et peut donc «filtrer» le dossier, devenu entre-temps volumineux, en fonction d'un problème déterminé.

A la demande de M. Huber, le Dr Müller lui remet le dossier des trois dernières années sur une clé USB. A son nouveau domicile, M. Huber se met à la recherche d'un médecin de famille qui puisse lire les données de sa clé USB et les reprendre dans le nouveau dossier électronique qu'il établira.

M. Huber trouve enfin ce médecin en 2015. Il s'agit d'un jeune médecin qui vient d'ouvrir son cabinet de médecine de famille.

tives peuvent, la plupart du temps, être transférées plus ou moins sans problème le cas échéant. En outre, les données comptables ne doivent plus pouvoir être transportées lors d'un changement de système.

Quelles mesures faut-il prendre pour que M. Huber puisse éventuellement trouver un nouveau médecin plus tôt?

L'échange de données n'a de sens que si les deux systèmes (la source et la cible) disposent des mêmes containers. Il est néanmoins quasiment impossible de tout uniformiser jusqu'au moindre détail. Et ce n'est d'ailleurs pas nécessaire. Mais après le transfert, les données saisies pour le status cardiaque devraient à nouveau figurer sous le status cardiaque et non pas sous le status dermatologique. Dans les propositions qui suivent, nous tenons uniquement à fixer le dénominateur commun et à montrer dans quelle direction il conviendrait de développer ce projet. 
Les données peuvent être transférées de $\mathrm{A}$ à $\mathrm{B}$ au moyen d'un schéma XML à définir [2].

Le mapping correspondant (c'est-à-dire la classification des contenus) permet d'exporter des données du système $\mathrm{A}$ au moyen d'un container de transfert pour les importer dans le système $\mathrm{B}$.

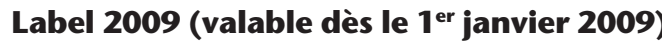
Centre de gravité: unification de la documentation, préparation de l'échange des données.

Les exigences de base suivantes doivent être remplies pour le dossier électronique du patient:

\section{Schéma SOAP}

Malgré le souhait de certains médecins d'avoir un dossier électronique doté d'une structure minimale, le schéma SOAP [3] peut être considéré comme le premier dénominateur commun, aussi $\mathrm{du}$ point de vue international. Le mot SOAP contient les quatre mots suivants: subjectivité, objectivité, assessment (= évaluation) et procédure. Lorsqu'un dossier contient ces quatre champs, on peut alors saisir une donnée selon la date ou le timbre dateur dans la banque de données en fonction de ce schéma. A partir de là, on peut en tout temps consulter les entrées et donc aussi les exporter.

\section{Liste des problèmes selon la classification ICPC-2}

Nous voulons et allons établir en Suisse le standard permettant de gérer la liste des problèmes et des diagnostics selon la classification ICPC-2 [4]. Le souci de conserver la vue d'ensemble existe aussi pour les dossiers de patients sur papier. Il n'en va pas autrement pour les dossiers électroniques, compte tenu du volume croissant des données. Mais si chacun établit la liste des problèmes selon son propre schéma, l'échange de données est impossible et la mise au courant au cabinet médical, p. ex. d'un médecin-assistant, d'un remplaçant ou justement d'un successeur, est entravée. Lorsque nous aurons réussi à gérer la liste des problèmes de manière uniforme, nous aurons fait un pas important en direction de la migration des données médicales.

3. Saisie des chiffres dans des champs séparés Les chiffres saisis pour un patient doivent être enregistrés dans des champs séparés.

Pour des raisons d'évaluation, un médecin a besoin de savoir en toute urgence où il doit enregistrer la valeur de la pression sanguine. Les valeurs concernant la pression sanguine, le pouls, la taille, le poids, le tour de taille, l'indice de masse corporelle, etc., sont des données à saisir judi- cieusement en tant que chiffres, donc de manière séparée et non pas dans le champ «Objectivité», bien qu'elles fassent naturellement partie des données objectives.

\section{Gestion des médicaments}

Les médicaments devraient être saisis sur la base du code utilisé en pharmacie ou d'un système analogue et avec le dosage selon le schéma 0 - 0 $0-0$. Un champ spécial «Intolérance à des médicaments» doit également être à disposition avec la saisie du motif.

\section{Données d'analyse de laboratoire}

Les données d'analyse de laboratoire sont également saisies avec le tampon dateur et les valeurs de référence concernées.

Cette liste est exhaustive pour le label 2009.

Les logiciels qui offrent les possibilités décrites ci-avant remplissent les exigences du label 2009. Il va de soi que les entreprises sont libres d'y intégrer de nouvelles fonctions, de remplir des exigences des futurs labels, de rendre possible des évaluations au moyen de graphiques, d'installer des interfaces techniques, etc.

\section{Label 2010 (valable dès le $\mathbf{1}^{\text {er }}$ janvier 2010)} Centre de gravité: RFE, échange ponctuel de données (upload).

Les éléments suivants viennent s'ajouter aux points 1 à 5 du label 2009:

\section{RFE (reason for encounter)}

En sus du schéma SOAP et de la liste des problèmes selon la classification ICPC-2, il est possible de saisir la consultation du point de vue du patient (reason for encounter) également selon la classification ICPC-2 dans un champ séparé. Le logiciel doit offrir cette option RFE. Au début, les médecins l'utiliseront intensivement et de manière très différente. La possibilité de saisir des consultations à différents niveaux est toutefois impérative pour des projets relevant de la médecine de famille.

\section{Echange de données (transfert du dossier du patient)}

L'utilisateur peut exporter les données des points 1 à 6 sans devoir faire appel à une firme de logiciels (schéma XML) et ces données peuvent être lues par un autre système.

Il est donc possible de transférer le dossier électronique du patient du médecin A au médecin $\mathrm{B}$ avec un archivage correct des contenus des points 1 à 6 à l'arrivée. 


\section{Téléchargement des données d'un PC sur un serveur (upload)}

A l'instar des données pour les centres fiduciaires, les données cliniques extraites à des fins de recherche peuvent être anonymisées et téléchargées sur un serveur défini (schéma XML).

L'utilisateur peut effectuer lui-même ce téléchargement sans devoir faire appel à une firme de logiciels.

Ces données proviennent des domaines des points 2 à 6 .

Label 2011 (valable dès le 1 $^{\mathrm{er}}$ janvier 2011) Centre de gravité: capacité de migrer, concept d'épisodes.

Le label 2010 doit être complètement rempli. Les éléments suivants viennent s'y ajouter:

\section{Concept des épisodes}

Le dossier électronique du patient est conçu de façon à reproduire le concept des épisodes à la base de la classification ICPC-2 [5]. Concrètement dit, il est désormais possible de saisir et de présenter les maladies de longue durée, la modification des problèmes pour le même épisode, etc.

\section{Exportation de la banque de données}

La banque de données est exportable en tant qu'un tout. Les contenus enregistrés sont exportables et importables dans une banque de données de code source libre (open-source, tel que postgres). La migration est effectuée par la firme de logiciels sans perte et sans support.

\section{Discussion}

En lançant une feuille de route pour le dossier électronique du patient en Suisse, nous émettons un signal clair pour l'échange des données et leur capacité à migrer.

Nous sommes convaincus que de nombreux médecins n'osent pas faire le pas en direction d'un cabinet libéré de la paperasserie, et ce en raison, principalement, de l'investissement élevé nécessaire et du manque d'échanges possibles. La possibilité d'échanger intégralement ou en partie un dossier électronique est échue depuis longtemps. Vu à long terme, il ne faut pas non plus qu'un médecin décide à la place de son éventuel successeur avec quel système celui-ci devra travailler un jour au cabinet médical.

Depuis le $1^{\text {er }}$ janvier 2008, les firmes de logiciels sont informées de cette situation et ont communiqué leur intérêt à coopérer. Le CD-ROM SGAM/SSMG.Informatics intitulé «Le dossier électronique du patient au cabinet médical» paraîtra cette année dans sa troisième édition. Sept firmes de logiciels ont promis de présenter des solutions avec vidéo d'écran sur le CD. Les scénarios actuels ont été complétés par deux nouveaux éléments: communication interne/gestion des données et intégration de la classification ICPC-2.

Quelques produits remplissent aujourd'hui déjà les exigences du label 2009.

Le site internet de la SSMG présentera à fin 2008 la liste des entreprises partenaires qui remplissent les critères du label, ce qui permettra aux médecins de savoir rapidement avec quel logiciel ils sont équipés, aussi pour le futur.

\section{Bibliographie}

1 Etude SISA FMH/SGAM.SSMG Informatics Janvier 2008.

2 L' «Extensible Markup Language»», abrégé XML, est un langage servant à hiérarchiser des données structurées sous forme de données de texte. Il est utilisé entre autres pour l'échange de données entre différents systèmes informatiques, spécialement paInternet. Les informations sont identifiées par des balises appelées tags (p.ex. <BDsyst $>160<$ /BDsyst $>$ ); elles peuvent alors être clairement attribuées.

3 Base provenant de: EQuiP Medical Record Project Team. Critères pour évaluer des dossiers électroniques de patients à l'aide d'une combinaison d'indicateurs de qualité permettant d'améliorer le dossier personnel. Zürich: compis; 2004.

4 WONCA-International Classification Committee WICC. www.globalfamilydoctor.com/wicc/.

5 Körner T, Saad A, Laux G, Rosemann T, Beyer M, Szecsenyi Joachim. Die Episode als Grundlage der Dokumentation. Dtsch Ärztebl. 2005;102(46): A 3168-72. 